\section{Breaking MYC metabolism}

\section{By Benjamin Boettner, Associate Editor}

The metabolic enzyme phosphoribosyl pyrophosphate synthetase 2 could represent a tractable way to modulate the notoriously undruggable oncogene MYC. ${ }^{1}$ The findings from a University of California, San Francisco team are licensed to Effector Therapeutics Inc. and provide a clue as to how the company plans to attack protein translation-related mechanisms in cancers.

The UCSF group now plans to identify specific inhibitors of phosphoribosyl pyrophosphate synthetase 2 (PRPS2) and wants to see if the mechanism is present in cancers promoted by oncogenes other than MYC (cMyc).

MYC is a transcription factor that tumors hijack to upregulate the metabolic machinery that promotes DNA and protein synthesis, glycolysis and glutaminolysis. ${ }^{2}$ The problem is that transcription factors are difficult to target
"Targeting cancer metabolism often is thwarted by redundancy and rewiring, and the fact that Prps2 is so differently regulated than Prps1 might allow less compensation."

-Neil Jones,

Cancer Research Technology
The synthetic lethality of Myc overexpression and Prps2 loss in mice was recapitulated by knocking down Prps2 using RNAi.

The reason healthy mice were not affected by the loss of Prps 2 is the presence of Prps1, which shares 95\% homology with Prps2. The key is that Prps1 is not affected by Myc and thus could carry out nucleotide biosynthesis. This also means that small molecule Prps2 inhibitors could provide tumor cell selectivity.

The findings were published in Cell. The team was led by Davide Ruggero, a professor in UCSF's Department of Urology.

"Classical high throughput screening has not offered a way to directly target MYC. These findings open up a new space for metabolic drug targets in MYC and perhaps other cancer contexts. As opposed to glycolysis and other metabolic pathways, direct regulators of nucleotide biosynthesis have not been as intensely looked at from a drug discovery perspective," said Neil Jones, senior group leader of discovery bioscience at Cancer Research Technology.

He added, "Targeting cancer metabolism often is thwarted by redundancy and rewiring, and the fact that Prps2 is so differently regulated than Prps1 might allow less compensation."

\section{Cause and Effector}

Ruggero is a cofounder of Effector Therapeutics, and the new paper is one of the first windows into the targets the company is attacking. UCSF has filed a patent on the findings, and the IP is licensed to Effector.

Effector raised $\$ 45$ million in a series A round last year. At the time, the company was not with small molecules or biologics. Indeed, there are only two disclosed molecules in development that target MYC, and both are nucleic acidbased therapies.

A team at UCSF set out to investigate whether protein synthesis and nucleotide synthesis were mechanistically linked downstream of MYC. The group began by asking which metabolic changes were tied to MYC's ability to upregulate protein biosynthesis. In a mouse model of Burkitt lymphoma, nucleotide levels in leukemic B cells were particularly dependent on Myc's ability to trigger protein translation. Additional studies showed that Myc-enhanced protein translation was directly coupled to nucleotide biosynthesis and that the rate-limiting enzyme was Prps2.

PRPS2 adds a pyrophosphate group from ATP to ribose-5-phosphate to generate a nucleotide precursor called 5-phosphoribosyl-1pyrophosphate (PRPP).

MYC was already known to transcriptionally elevate levels of eukaryotic translation initiation factor $4 \mathrm{E}$ (eIF4E), which in turn binds to enhancers in target mRNAs to upregulate their translation. The UCSF team's studies identified the same enhancer element in the $5^{\prime}$ portion of the Prps2 mRNA, thus providing a mechanism for its regulation by Myc.

Importantly, genetic knockout of $\operatorname{Prps} 2$ by itself had no physiological or metabolic consequences in mice. However, in the presence of hyperactive Myc, loss of Prps2 became lethal-it dramatically elevated apoptosis of B lymphocytes in the Myc-driven lymphoma model. discussing its technology, except to say it was developing small molecules that selectively regulate translation of oncogenes.

Effector did not return calls seeking comment

Ruggero now wants to evaluate the findings in a larger collection of tumor models driven by Myc and other oncogenes.

Zuzana Zachar, director of research at Cornerstone Pharmaceuticals Inc., said that validating PRPS2 as a target will require a better understanding of its role in normal, nontransformed cells. "The lack of gross phenotypic abnormalities in mice does not exclude that Prps 2 could perform functions in response to challenges like wounding, infection or involving inflammation," she said.

Cornerstone's $\mathrm{CPI}-613$, a pyruvate dehydrogenase (PDH) inhibitor that interferes with cancer carbohydrate metabolism, is in Phase II testing for multiple cancers.

Both Jones and Heather Christofk said that the cancer cell lethality caused by Myc hyperactivation and Prps 2 depletion should be validated in other tumor models that rely on Myc activation, such as some forms of breast cancer.

"An immediate next step to validate PRPS2 is to determine whether its levels are elevated in human cancers and whether this tracks with MYC overexpression or other oncogenes known to result in eIF4E activity," said Christofk, an assistant professor at the Institute for Molecular Medicine at the University of California, Los Angeles David Geffen School of Medicine. 


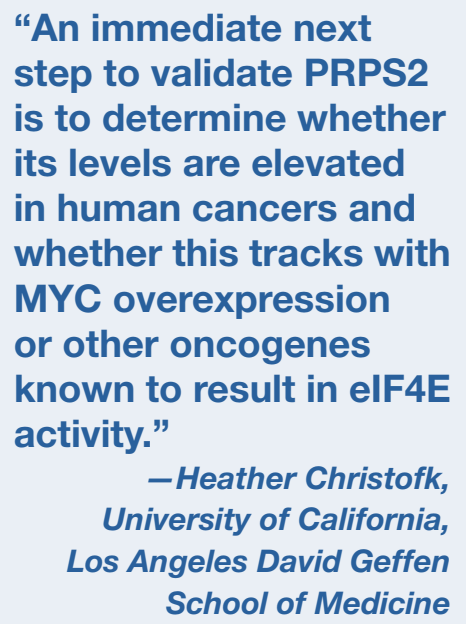

"An immediate next step to validate PRPS2 is to determine whether its levels are elevated in human cancers and whether this tracks with MYC overexpression or other oncogenes known to result in eIF4E activity."

- Heather Christofk, University of California, Los Angeles David Geffen School of Medicine

"Other oncogenic pathways could impinge on the same translational enhancer in the Prps2 mRNA, and it could be part of a larger signature," added Jones.

Ruggero told SciBX that his laboratory is starting to investigate whether the Prps2-based mechanism is conserved in an array of Myc-dependent and Mycindependent tumor models.

\section{Inhibitor search}

Ruggero's team at UCSF is collaborating with Kevan Shokat's lab to identify PRPS2-specific inhibitors. Shokat, also a cofounder of Effector, is chair of the Department of Cellular and Molecular Pharmacology at UCSF and a Howard Hughes Medical Institute investigator.

One key to finding an inhibitor will be getting selectivity for PRPS2 over PRPS1.

"Selective inhibitors of closely related isozymes are challenging to identify. However, lessons from the PI3K [phosphoinositide 3-kinase] field and many other enzyme classes have taught that selective inhibition of a single isozyme is possible with careful and diligent medicinal chemistry," said Shokat. "This can be greatly aided by structure-based design."

Marion Dorsch, VP of biology at cancer metabolism company Agios Pharmaceuticals Inc., agreed that elucidating the structures of PRPS1 and PRPS2 could help guide the design of selective inhibitors. The comparison of the 3D structures of PRPS1 and PRPS2 may reveal exploitable differences between the two enzymes despite the $95 \%$ amino acid sequence identity.

"High throughput screening could enhance the probability to identify isoform-selective small molecules with allosteric inhibitory properties. Also, looking for inhibitors acting upstream to modulate PRPS2 vs. PRPS1" could be an approach worth exploring, she said.

Boettner, B. SciBX 7(24); doi:10.1038/scibx.2014.691

Published online June 19, 2014

REFERENCES

1. Cunningham, J.T. et al. Cell; published online May 22, 2014; doi:10.1016/j.cell.2014.03.052

Contact: Davide Ruggero, University of California, San Francisco, Calif. e-mail: davide.ruggero@ucsf.edu

2. Dang, C.V. Genes Cancer 1, 526-531 (2010)

COMPANIES AND INSTITUTIONS MENTIONED

Agios Pharmaceuticals Inc. (NASDAQ:AGIO), Cambridge, Mass. Cancer Research Technology, London, U.K. Cornerstone Pharmaceuticals Inc., Cranbury, N.J. Effector Therapeutics Inc., San Diego, Calif. Howard Hughes Medical Institute, Chevy Chase, Md. University of California, San Francisco, Calif. University of California, Los Angeles David Geffen School of Medicine, Los Angeles, Calif. 